\title{
Tanshinone IIA Inhibits High Glucose- Induced Collagen Synthesis via Nuclear Factor Erythroid 2-Related Factor 2 in Cardiac Fibroblasts
}

\author{
Yi-Ting Tsaia Shih-Hurng Lohe, ${ }^{a, c, d} \quad$ Chung-Yi Lee ${ }^{b} \quad$ Shiao-Ping Lee \\ Yen-Lin Chen ${ }^{f} \quad$ Tzu-Hurng Cheng ${ }^{c, g}$ Chien-Sung Tsaib,
}

${ }^{a}$ Graduate Institute of Medical Sciences, National Defense Medical Center, Taipei, ${ }^{b}$ Division of Cardiovascular Surgery, Department of Surgery, Tri-Service General Hospital, National Defense Medical Center, Taipei, 'Department of Pharmacology, National Defense Medical Center, Taipei, dDepartment of Pharmacy Practice, Tri-Service General Hospital, National Defense Medical Center, Taipei, eDivision of Oral and Maxillofacial Surgery, Department of Dentistry, School of Dentistry, Tri-Service General Hospital and National Defense Medical Center, Taipei, fDepartment of Radical Diagnostic, Taoyuan Armed Forced General Hospital, Taoyuan, 9Department of Biochemistry, School of Medicine, College of Medicine, China Medical University, Taichung, Taiwan

\section{Key Words}

Tanshinone IIA - High Glucose - Cardiac Fibroblast - Collagen Synthesis - Nuclear Factor Erythroid 2-related Factor 2

\begin{abstract}
Background/Aims: Diabetes is associated with increased incidence of myocardial dysfunction, which is partly characterized by interstitial and perivascular fibrosis. Cardiac fibroblasts have been identified as an important participant in the development of cardiac fibrosis. Exposure of cultured cardiac fibroblasts to high glucose resulted in increased collagen synthesis. Tanshinone IIA can alleviate the ventricular fibrosis that develops in a number of different experimental conditions. However, whether tanshinone IIA can prevent high glucoseinduced collagen synthesis in cardiac fibroblasts remains unknown. The aim of this study was to evaluate the effects of tanshinone IIA on high glucose-induced collagen synthesis in cardiac fibroblasts. Methods: Rat cardiac fibroblasts were cultured in high glucose $(25 \mathrm{mM})$ media in the absence or presence of tanshinone IIA and the changes in collagen synthesis, transforming growth factor- $\beta 1$ (TGF- $\beta 1$ ) production and related signaling molecules were assessed by ${ }^{3} \mathrm{H}$-proline incorporation, quantitative polymerase chain reaction, enzyme linked immunosorbent assay, and Western blotting. Results: The results indicate cardiac fibroblasts exposed to high glucose condition show increased cell proliferation and collagen synthesis and these effects were abolished by tanshinone IIA treatment. Furthermore, the inhibitory
\end{abstract}

Yi-Ting Tsai, MD Department of Surgery, Tri-Service General Hospital National Defense Medical Center, Taipei 114 (Taiwan) Tel. 886-2-87927376 E-Mail cvsallen@ndmctsgh.edu.tw 


\section{Cellular Physiology Cell Physiol Biochem 2018;51:2250-2261

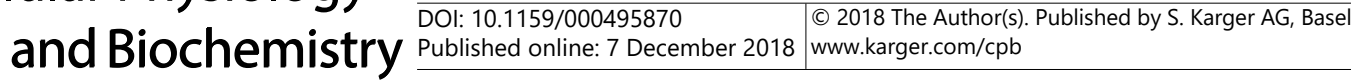 \\ Tsai et al.: Tanshinone IIA Inhibits Collagen Synthesis}

effect of tanshinone IIA on high glucose induced cell proliferation and collagen synthesis may be associated with its activation of the nuclear factor erythroid 2-related factor 2 (Nrf2) and the inhibition of TGF- $\beta 1$ production and Smad2/3 phosphorylation. Conclusion: In summary, our results highlights the critical role tanshinone IIA plays as an antioxidant in attenuating high glucose-mediated collagen synthesis through inhibiting TGF- $\beta 1 / \mathrm{Smad}$ signaling in cardiac fibroblasts which provide a mechanistic basis for the clinical application of tanshinone IIA in the treating diabetic-related cardiac fibrosis.

(c) 2018 The Author(s)

Published by S. Karger AG, Basel

\section{Introduction}

Tanshinone IIA was extracted from Danshen, a popular medicinal herb which has been used in traditional Chinese medicine in treating cardiovascular diseases for many years [1]. A series of our studies demonstrated tanshinone IIA prevents doxorubicininduced cardiomyocyte apoptosis [2], attenuates $\mathrm{H}_{2} \mathrm{O}_{2}$-induced injury [3] and induces heme oxygenase-1 (HO-1) expression by activating the nuclear factor erythroid 2-related factor 2 (Nrf2) pathway in vascular endothelial cells [4], inhibits angiotensin II-induced cell proliferation via antioxidant activity in rat cardiac fibroblasts [5]. However, the effects and mechanisms of tanshinone IIA on cardio-protection are yet to be delineated.

Diabetes mellitus (DM) is one of the leading causes of cardiovascular diseases and myocardial fibrosis (characterized by the proliferation of cardiac fibroblasts and abundant accumulation of matrix proteins in the extracellular space) has been previously described as one of the major pathological consequences of long-standing DM in diabetic hearts [6]. High glucose (or hyperglycemia), the main feature of DM, has been shown to stimulate cardiac fibroblast proliferation in vitro and increase fibroblast collagen production [7], contributing to the pathological changes in the cardiovascular system. Transforming growth factor- $\beta 1$ (TGF- $\beta 1$ ) is a pro-sclerotic cytokine consistently implicated in cardiac fibrosis and its expression is enhanced by diabetes [7]. In addition, high glucose mediates increased TGF- $\beta 1$ activity and downstream canonical Smad signaling with tanshinone IIA-containing capsules inhibiting fibrosis and improving cardiac function via suppression of this pathway [8].

However, no study has directly addressed the effects of tanshinone IIA on high glucoseinduced collagen synthesis in cardiac fibroblasts. The aims of this study were to investigate the effect of tanshinone IIA on high glucose-induced collagen synthesis and identify whether the underlying mechanisms are associated with its antioxidant effects and the modulation of the TGF- $\beta 1 /$ Smad signaling pathway in cardiac fibroblasts.

\section{Materials and Methods}

\section{Materials}

Dulbecco's modified Eagle's medium (DMEM), fetal calf serum, and tissue culture reagents were from Invitrogen/GIBCO (Grand Island, NY, U.S.A). Tanshinone IIA (purchased from Santa Cruz Biotechnology) was dissolved in dimethyl sulfoxide (DMSO), and the DMSO content in all groups was $0.1 \%$. The primary antibodies (anti-p-Smad2/3, anti-Smad2/3, anti-GAPDH, anti-PARP) were purchased from Cell Signaling Technology (Boston, MA, USA) and the primary antibodies (anti-HO-1, anti-Nrf2) were purchased from Santa Cruz Biotechnology (Santa Cruz, CA, USA). All other reagent-grade chemicals were purchased from the Sigma-Aldrich Chemical Co. (St. Louis, MO, U.S.A.).

\section{Culture of cardiac fibroblasts}

The investigation conformed to the Guide for the Care and Use of Laboratory Animals published by the US National Institutes of Health (NIH Publication No. 85-23, revised 1996) and was also approved by the Institutional Animal Care and Use Committee of China Medical University (IACUC 2016-032). Primary cultures of neonatal rat cardiac fibroblasts were prepared as previously described [9]. Briefly, ventricles from 1- to 2-day-old neonatal Sprague-Dawley rats were cut into chunks of approximately $1 \mathrm{~mm}^{3}$ by using 


\section{Cellular Physiology Cell Physiol Biochem 2018;51:2250-2261

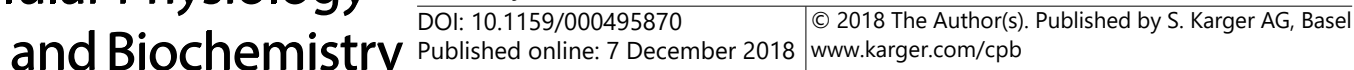 \\ Tsai et al.: Tanshinone IIA Inhibits Collagen Synthesis}

scissors and were subjected to trypsin (0.125\%; Invitrogen, Carlsbad, CA, U.S.A.) digestion in phosphate buffered saline (PBS). Dispersed cells were incubated on 100 -mm culture dishes for $30 \mathrm{~min}$ in a $5 \% \mathrm{CO}_{2}$ incubator. Neon myocytes attached to the bottom of the dishes were subsequently incubated with DMEM supplemented with $10 \%$ fetal calf serum for an additional 2 to $4 \mathrm{~d}$. Confluent neon myocytes were treated with trypsin and subcultured. Subconfluent ( $70 \%$ confluency) cardiac fibroblasts grown in culture dishes from the second to fourth passage were used in the experiments and were $>99 \%$ positive for vimentin antibodies (Sigma-Aldrich). Serum-containing medium from the cultured cells was replaced with serumfree medium, and the cells were then exposed to the agents as indicated. Note that for the fibroblast proliferation assay and the TGF-beta1 secretion assay, following incubation with Tanshinone IIA for $12 \mathrm{~h}$, cardiac fibroblasts were then exposed to a serum-free normal glucose medium (i. e. $5.6 \mathrm{mM}$ glucose) or high-glucose medium (i. e. $25 \mathrm{mM}$ glucose) for another $24 \mathrm{~h}$ before the analyses.

\section{Proliferation assay}

Proliferation was assessed by quantifying 5-bromo-2'-deoxyuridine (BrdU) incorporation [5]. Cells were removed from the culture dish by addition of trypsin and then centrifuged. Cell proliferation was assessed by the incorporation of BrdU. Cells $\left(1 \times 10^{4}\right.$ cells/well $)$ were incubated in 96 -well plastic plates. Then, BrdU $(10 \mu \mathrm{M})$ was added to the medium and the cells were incubated for another $18 \mathrm{~h}$. Subsequently, the cells were fixed and BrdU incorporation was determined with a Cell Proliferation enzyme-linked immunosorbent assay (ELISA) Kit (Roche Diagnostics, Mannheim, Germany) according to the manufacturer's instructions.

\section{${ }^{3} \mathrm{H}$-proline incorporation assay}

For stimulation experiments, high glucose $(25 \mathrm{mM})$ with or without tanshinone IIA was used. Cardiac fibroblasts were then incubated with ${ }^{3} \mathrm{H}$-proline $\left(1 \mathrm{mCi} /\right.$ well, L- $\left[2,3,4,5-{ }^{3} \mathrm{H}\right]$-proline; Amersham Biosciences, Buckinghamshire, UK) for $24 \mathrm{~h}$. Incorporation of exogenous ${ }^{3} \mathrm{H}-$ proline was determined by scintillation counting as previously described [9]. In order to rule out the interference of osmolality, we actually had performed a pilot study to determine the effects of osmotic pressure on cardiac fibroblasts. The effect on three groups including normal control group (medium with $5.6 \mathrm{mM}$ glucose), high glucose treatment group (medium with $25 \mathrm{mM}$ glucose), and high osmolality group (medium with $5.6 \mathrm{mmol} / \mathrm{L}$ glucose $+19.4 \mathrm{mM}$ mannitol) had been observed. When cardiac fibroblasts were treated for $24 \mathrm{~h}$ with high glucose medium, significant increases in cell proliferation and [3H]proline incorporation occurred. These effects were not observed in high-osmolarity-treated cardiac fibroblates, which rules out the interference of osmotic pressure changes on the Tanshinone IIA-induced results.

\section{$R N A$ extraction and quantitative polymerase chain reaction (Q-PCR) analysis}

Total RNA was extracted from cardiac fibroblasts using the TRIzol method according to the protocol recommended by the manufacturer (Invitrogen, Carlsbad, CA, USA), and used to synthesize single-stranded complementary (c) DNA with a High-Capacity cDNA Reverse Transcription Kit (Applied Biosystems, Foster City, CA, USA) as previously described [5]. TGF- $\beta 1$ mRNA was quantified using TaqMan Gene Expression Master Mix (Applied Biosystems) with specific primers in an ABI 7300 Real-Time PCR System (Applied Biosystems). TaqMan Gene Expression Assay kits containing specific primers for TGF- $\beta 1$ (lot no. Rn00572010_m1), and GAPDH (lot no. Rn01775763_g1) were obtained from Applied Biosystems. The specific primers for GAPDH were used to normalize the amount of sample added. All quantifications were performed in triplicate samples for three separate experiments.

\section{Measurement of TGF- $\beta 1$ concentrations}

TGF- $\beta 1$ levels were measured in culture medium using a commercial enzyme-linked immunosorbent assay (ELISA) kit (R\&D Systems Inc., Minneapolis, MN, USA) according to the manufacturer's protocols. Results were normalized to cellular protein content in all experiments, and expressed as a percentage relative to the control group. 


\section{Cellular Physiology Cell Physiol Biochem 2018;51:2250-2261 and Biochemistry \begin{tabular}{l|l} 
DOI: 10.1159/000495870 & (c) 2018 The Author(s). Published by S. Karger AG, Basel \\
www.karger.com/cpb
\end{tabular} \\ Tsai et al.: Tanshinone IIA Inhibits Collagen Synthesis}

\section{Western blot analysis}

Nuclear protein was prepared as previously described [4]. Western blot analysis was performed as previously described [5]. Whole-cell extracts were obtained in a RIPA buffer (10 mM Tris, pH 7.5, $150 \mathrm{mM}$ $\mathrm{NaCl}, 0.1 \%$ SDS, $1.0 \%$ Triton X-100, 1\% Deoxycholate, 5 mM EDTA, 1 mM sodium orthovanadate, 1 mM PMSF, and protease inhibitor cocktail; Complete, Roche Diagnostics GmbH, Germany). Extracts or proteins were separated by SDS-PAGE followed by electrotransfer to polyvinylidene difluoride (PVDF) membranes and probed with antisera, followed by horseradish peroxidase-conjugated secondary antibodies. The proteins were visualized by chemiluminescence, according to the manufacturer's instructions (Pierce Biotechnology Inc., IL, USA). Images were quantified by densitometry. The results were normalized to GAPDH (or PARP). All experiments were repeated for 3 times.

\section{Flow cytometric assay of 2',7'-dichlorodihydrofluorescein oxidation}

The determination of intracellular reactive oxygen species production was based on the oxidation of $2^{\prime}, 7^{\prime}$-dichlorodihydrofluorescein (DCFH) to a fluorescent $2^{\prime}, 7^{\prime}$-dichlorofluorescein (DCF) as previously described [5]. DCFH was added at a final concentration of $10 \mu \mathrm{M}$ and incubated for $30 \mathrm{~min}$ at $37^{\circ} \mathrm{C}$. The cells were then washed once with PBS and maintained in a 1-ml culture medium. Following drug treatment, the medium was aspirated and cells were washed twice with PBS, and then dissociated with trypsin. Cellular fluorescence was determined by flow cytometry (FACS-SCAN, Becton-Dickinson, Franklin Lakes, NJ, USA). Cells were excited with an argon laser at $488 \mathrm{nM}$, and measurements were taken at 510-540 nm.

\section{The Small Interfering RNA Targeting of Nrf2}

The small interfering RNA targeted to Nrf2 (Nrf2-siRNA, sc-37030) and control siRNA (Scramble siRNA) were purchased from Santa Cruz Biotechnology, Inc. (Dallas, TX, USA). Cardiac fibroblasts were transfected with 100nM Nrf2-siRNA or Control siRNA with Lipofectamine 2000 (Invitrogen) for 24 hours according to the manufacturer' protocol. The silencing effects were confirmed by Western blot.

\section{Statistical Analysis}

Results are expressed as mean \pm S.E.M. for at least six experiments unless designated otherwise. Statistical analysis was performed using Student's t test or analysis of variance (ANOVA) followed by Tukey's multiple comparisons using GraphPad Prism (GraphPad Software, San Diego, CA, USA). A value of $P<0.05$ was considered to be statistically significant.

\section{Results}

Tanshinone IIA inhibits high glucose induced fibroblast proliferation and collagen synthesis We first tested the role of tanshinone IIA on cardiac fibroblast proliferation and collagen synthesis. Isolated cardiac fibroblast cells were cultured in normal and high glucose medium. The effects of tanshinone IIA on high glucose-stimulated rat cardiac fibroblast proliferation and collagen synthesis were assessed with both BrdU and ${ }^{3} \mathrm{H}$-proline incorporation. Compared with the normal glucose medium, high glucose $(25 \mathrm{mM})$ for $24 \mathrm{~h}$ slightly stimulated fibroblast proliferation and collagen synthesis (Fig. 1A and 1B). Pretreatment of cardiac fibroblasts with tanshinone IIA $(10$ and $30 \mu \mathrm{M})$ for 30 min followed by exposure to high glucose resulted in a significant decrease in high glucose-increased cell proliferation and collagen synthesis (Fig. 1A and 1B). These data clearly suggest tanshinone IIA inhibited high glucose-induced proliferation and collagen synthesis in rat cardiac fibroblasts.

Tanshinone IIA inhibits high glucose induced TGF- $\beta 1$ expression and Smad2/3 phosphorylation in cardiac fibroblasts

Cytokine TGF- $\beta 1$ has been found to influence each of the single components of the remodeling process and is a key mediator of pathological cardiac fibrosis [10]. To investigate whether tanshinone IIA affects TGF- $\beta 1$ in cardiac fibroblasts exposed to high glucose medium, cardiac fibroblasts were treated with tanshinone IIA under high glucose conditions. The expression of TGF- $\beta 1$ was determined by QPCR and ELISA. As shown in Fig. 2A, cardiac 
fibroblasts treated with high glucose medium showed increased mRNA expression of TGF- $\beta 1$ when compared with cardiac fibroblasts in the normal group $(5.6 \mathrm{mM}$ glucose). However, high glucose medium induced TGF- $\beta 1$ upregulation was prevented by treatment of cardiac fibroblasts with tanshinone IIA (10 and 30 $\mu \mathrm{M})$. ELISA assay results revealed the content of TGF- $\beta 1$ in cardiac fibroblasts supernatant was elevated by high glucose medium. Similarly, the promotion effects of high glucose medium on TGF- $\beta 1$ secretion in cardiac fibroblasts were reversed by tanshinone IIA (10 and $30 \mu \mathrm{M}$ )

Fig. 2. Tanshinone IIA inhibits high glucose-induced TGF- $\beta 1$ expression and Smad2/3 phosphorylation in cardiac fibroblasts. Results were shown as mean \pm S.E.M. $(\mathrm{n}=6) .{ }^{*} \mathrm{P}<0.05$ versus normal glucose control group; \# $\mathrm{P}<0.05$ versus high glucose group. (A) Cardiac fibroblasts were treated with normal glucose (NG) medium or high glucose (HG) medium in the absence or the presence of tanshinone IIA $(1,3$, 10 or $30 \mu \mathrm{M}$ ) for $24 \mathrm{~h}$, the mRNA expression levels of TGF- $\beta 1$ was detected by Q-PCR with GAPDH as the normalization control. (B) The secretion level of TGF- $\beta 1$ in cardiac fibroblasts supernatant was measured by ELISA assay after cardiac fibroblasts were treated with HG medium in the absence or presence of tanshinone IIA $(1,3,10$ or $30 \mu \mathrm{M}$ ) for $24 \mathrm{~h}$. (C) The protein expression levels of $\operatorname{Smad} 2 / 3$, and P-Smad2/3 were detected by Western blotting, NG medium containing human recombined TGF- $\beta 1(10 \mathrm{ng} / \mathrm{mL})$ was used as a positive control. Cardiac fibroblasts were treated with NG medium or HG medium in the absence or presence of tanshinone IIA $(10 \mu \mathrm{M})$ for $2 \mathrm{~h}$. Representative micrograph of expression of Smad2/3 and P-Smad2/3 in Western blot analysis (left) and quantitative results (right).
Fig, 1. Effects of tanshinone IIA on high glucose-induced cell proliferation and collagen synthesis in cardiac fibroblasts. Cardiac fibroblasts were cultured in a serum-free normal glucose medium (NG, $5.6 \mathrm{mM}$ glucose) or high glucose medium (HG, 25 mM glucose) in the absence or presence of 10 or $30 \mu \mathrm{M}$ ) for $24 \mathrm{~h}$. Results were shown as mean \pm S.E.M. (n=6). ${ }^{*} \mathrm{P}<0.05$ versus

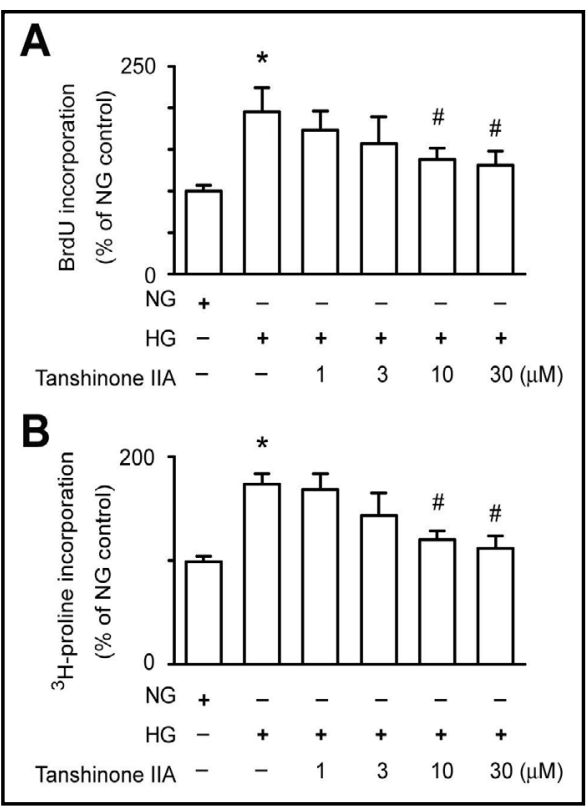

NG control group; \# $\mathrm{P}<0.05$ versus HG group. (A) Cell proliferation was assessed using the BrdU incorporation. (B) Collagen synthesis was assessed using ${ }^{3} \mathrm{H}$-proline incorporation. tanshinone IIA $(1,3$,

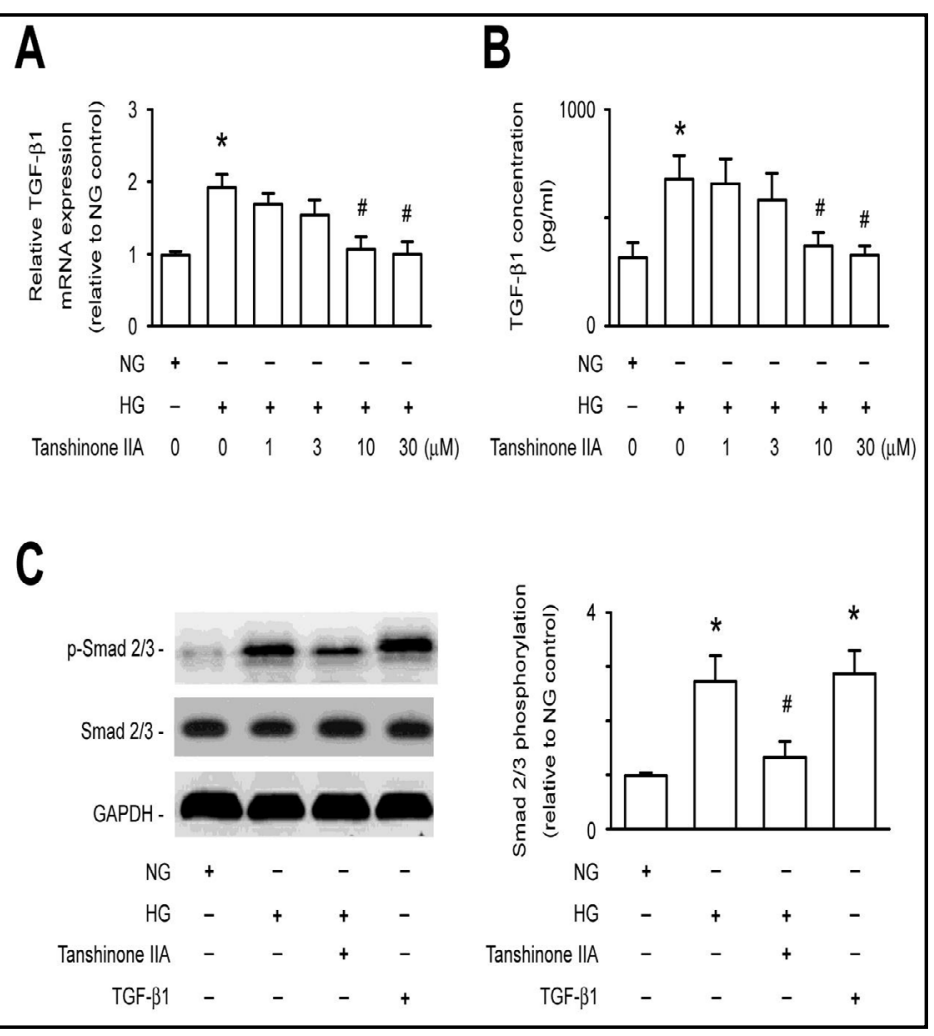


treatment (Fig 2B).

TGF- $\beta$ receptors

exert many of their

effects through Smad- $2 / 3$

phosphorylation [10].

Therefore, downstream of

TGF- $\beta 1$, phosphorylated

Smad2/3 was also detected.

Human recombined TGF- $\beta 1$

was used as a positive

control. After treatment for

$24 \mathrm{~h}$, total cell proteins were extracted and subjected to Western blotting assay. As illustrated in Fig. 2C, high glucose medium treatment for $2 \mathrm{~h}$ can enhance the phosphorylation level of Smad2/3. However, high glucose medium stimulated Smad2/3 phosphorylation was suppressed by tanshinone IIA $\quad(10$ $\mu \mathrm{M})$ treatment. These observations indicated tanshinone IIA may inhibit the high glucose mediated fibrosis response through regulating the TGF- $\beta 1 /$ Smad pathway.

Tanshinone IIA lessens high glucose induced ROS production and collagen synthesis in cardiac fibroblasts Increased reactive oxygen species (ROS) has been demonstrated to play an important role in the development of cardiac fibrosis in the setting of diabetes [11]. We next explored the mechanism by which tanshinone IIA inhibited the high glucose-induced collagen synthesis, mainly focusing on ROS generation. The fluorescence intensity of ROS probe DCFH-DA was detected by a flow cytometer in response to the levels of ROS. N-acetylcysteine (NAC) was selected for its common use as a well-known antioxidant of ROS measurement [11]. Our data showed the fluorescence intensity enhanced by high glucose was significantly reduced by tanshinone IIA $(10 \mu \mathrm{M})$ (Fig. $3 \mathrm{~A})$. Pre-treatment with NAC ( $5 \mathrm{mM})$ for $2 \mathrm{~h}$ also remarkably alleviated the ROS triggered by high glucose medium. Further, we tested the role of tanshinone IIA and NAC on cardiac fibroblast proliferation and collagen synthesis. Compared with the normal glucose medium, high glucose stimulated fibroblast proliferation was significantly blocked by tanshinone IIA or NAC treatment (Fig. 3B). Besides the pro-proliferative role of high glucose on cardiac fibroblasts, tanshinone IIA or NAC also attenuated the collagen synthesis induced by high glucose (Fig. 3C). These findings suggested the anti-fibrotic effect of tanshinone IIA might be related to the reduced oxidative stress level.
Fig. 3. Tanshinone IIA inhibits high glucose-induced ROS in cardiac fibroblasts. as mean \pm S.E.M. $(\mathrm{n}=6) . \quad * \mathrm{P}<0.05$ versus normal gucose (NG) control versus high glucose (HG) group. (A) Effects of tanshinone IIA on HG-induced ROS generation. Cardiac fibroblasts were cultured in $\mathrm{NG}$, or HG medium for 30 min, or preincubated with tanshinone (IA $(10 \mu \mathrm{M})$ or NAC (5mM) for $30 \mathrm{~min}$ and then stimulated 30 min Column graph of mean cell fluorescence for DCF. The fluorescence intensities in NG control cells are (B) Effects of tanshinone IIA and NAC on HG induced fibroblast proliferation. (C) Effects of tanshinone IIA and NAC on HG induced collagen synthesis. Cardiac fibroblast cells were cultured in NG or HG in the absence or presence of tanshinone IIA $(10 \mu \mathrm{M})$ or NAC $(5 \mathrm{mM})$ for $24 \mathrm{~h}$. 


\section{Cellular Physiology Cell Physiol Biochem 2018;51:2250-2261 and Biochemistry \begin{tabular}{l|l} 
DOI: 10.1159/000495870 & (c) 2018 The Author(s). Published by S. Karger AG, Basel \\
www.karger.com/cpb
\end{tabular}

Tanshinone IIA activates Nrf2 signaling pathway

Nrf2 is a well-known anti-oxidative protein which is also frequently related to anti-fibrotic actions [12, 13]. To better understand the possible signaling pathways and mechanisms implicated in the action of tanshinone IIA, we detected the expression of Nrf2 and Nrf2 downstream HO-1 in cardiac fibroblasts under high glucose conditions. Our data showed a high glucose condition could induce a slightly elevation of the translocation of Nrf2 from the cytoplasm to the nucleus as well as by enhance HO-1 expression, the effect of which was further augmented by tanshinone IIA treatment (Fig. 4A and 4B). These data suggested a role of the Nrf2/HO-1 signaling pathway in the anti-fibrotic effects of tanshinone IIA.

Tanshinone IIA suppresses high glucose induced TGF- $\beta 1$ expression, fibroblast proliferation and collagen synthesis via Nrf2 signaling

To further verify the role of Nrf2 in the anti-fibrotic effect induced by tanshinone IIA, Nrf2 small silenced RNA (siRNA) were used to determine the role of Nrf2 in the effects of tanshinone IIA on high glucose induced TGF- $\beta 1$ expression, cardiac fibroblast proliferation and collagen synthesis. Cells were transfected with Nrf2 siRNA, followed by treatment with $10 \mu \mathrm{M}$ tanshinone IIA for $12 \mathrm{~h}$ and then treating the cells with high glucose for $24 \mathrm{~h}$. Transfected cells with Nrf2 siRNA almost totally blocked the Nrf2 expression (data not shown). The levels of TGF- $\beta 1$ expression significantly increased under high glucose conditions as compared to the normal glucose control group (Fig. 5A). However, these increases were inhibited by tanshinone IIA. In contrast, silencing Nrf2 by siRNA partially abolished tanshinone IIA's effect. As expected, tanshinone IIA significantly decreased high glucose induced fibroblast proliferation and collagen synthesis, which was also partially reversed by Nrf2 siRNA (Fig. 5B and 5C). Collectively, these data indicated tanshinone IIA inhibited TGF- $\beta 1$ pathway activation and the proliferation and collagen synthesis of cardiac fibroblasts under high glucose condition in an Nrf-2 dependent manner.

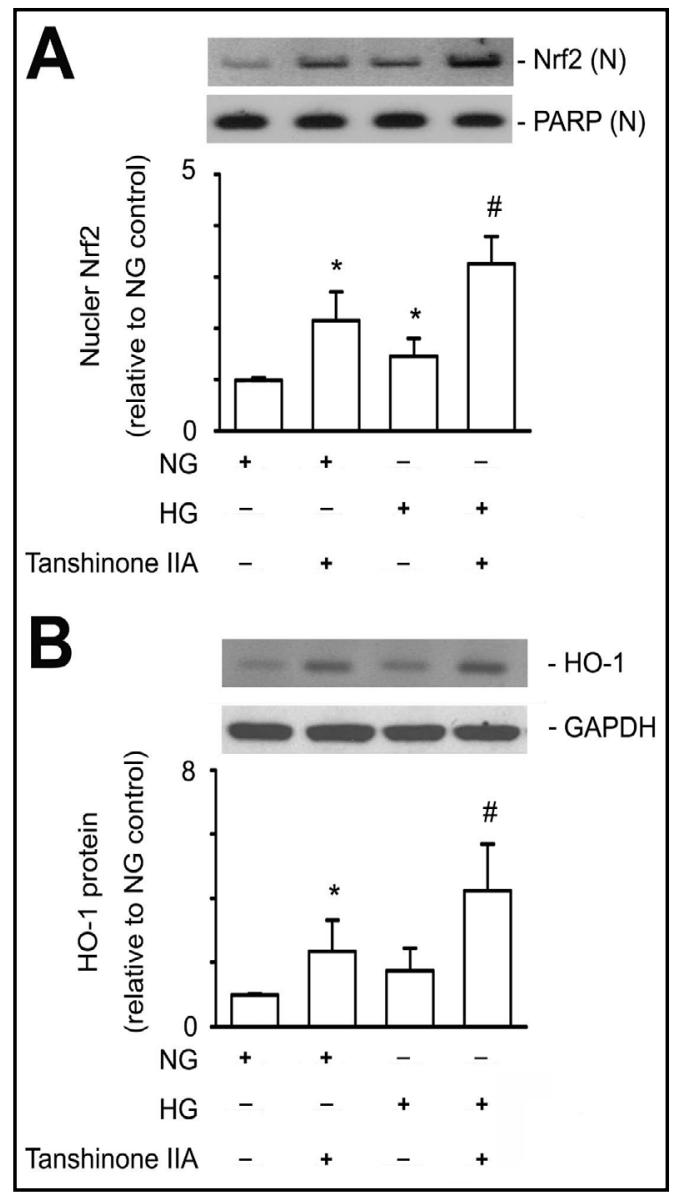

Fig. 4. Tanshinone IIA activates Nrf2 signaling pathway in cardiac fibroblasts. The relative protein expressions of nuclear Nrf2 to PARP, and HO-1 to GAPDH were expressed in the bar graphs. Results were shown as the mean \pm S.E.M. $(\mathrm{n}=4) .{ }^{*} \mathrm{P}<0.05$ vs. normal glucose control group. \# indicates a $\mathrm{P}$ value $<0.05$ compared with the high glucose group. (A) Effect of tanshinone IIA on Nrf2 translocation. Cells were treated with tanshinone IIA $(10 \mu \mathrm{M})$ or not for $12 \mathrm{~h}$, followed by normal glucose (NG) or high glucose (HG) for $12 \mathrm{~h}$. N, nuclear extract. (B) Quantification of the expression of HO-1 was detected by Western blot. Cells were pretreated with tanshinone IIA (10 $\mu \mathrm{M}$ ) or not for $12 \mathrm{~h}$, followed by NG or HG for $24 \mathrm{~h}$. 


\section{Discussion}

Cardiac fibrosis is one of the major pathological processes in diabetes, characterized by the proliferation of cardiac fibroblasts and excessive accumulation of extracellular matrix proteins within the interstices of the myocardium [6]. Hyperglycemia (high glucose) is a manifestation presented in DM [7]. It has been reported high glucose stimulates fibroblast proliferation [14], activates the transcription and secretion of extracellular matrix proteins [15], and transduces fibrogenic signals through ROS generation [16]. In addition, pro-inflammatory cytokines and chemokines may recruit fibrogenic leukocyte subsets in the cardiac interstitium. TGF- $\beta$ induction in diabetic hearts may involve the direct actions of high glucose [17]. Canonical TGF- $\beta 1$ signaling involves the receptor activated Smad2/3 proteins (Smad2 and Smad3), which upon phosphorylation, translocate to the nucleus and act as transcription factors [18]. Activation of TGF- $\beta /$ Smad signaling may activate fibroblasts, inducing deposition of structural extracellular matrix proteins and matricellular macromolecules [7]. In the present study, our results indicated high glucose condition induced upregulation of TGF- $\beta 1$ and activation of TGF- $\beta 1 /$ Smad pathway may be involved in high glucose mediated collagen production in cardiac fibroblasts. These results indicated controlling high glucose may have important therapeutic implications in preventing and treating diabetic-related cardiac fibrosis.

Tanshinone IIA is the liposoluble active ingredient of the traditional Chinese medical plant S. miltiorrhiza. Recent studies indicated tanshinone IIA may have potent anti-fibrotic effects on the pathological fibrosis process in different organs [19-21]. It has been reported that a tanshinone IIA-containing capsule can alleviate the myocardial fibrosis caused by streptozocin-induced diabetes in rats with high a fat-diet [8], probably by inhibiting the proliferation of cardiac fibroblasts [5], and by reducing the production of extracellular matrix proteins [21]. In another study, tanshinone IIA exhibited an antifibrotic effect through reducing inflammatory mediators and increasing antioxidant enzymes in a rat model of cirrhosis [22]. Based on these previously published data,

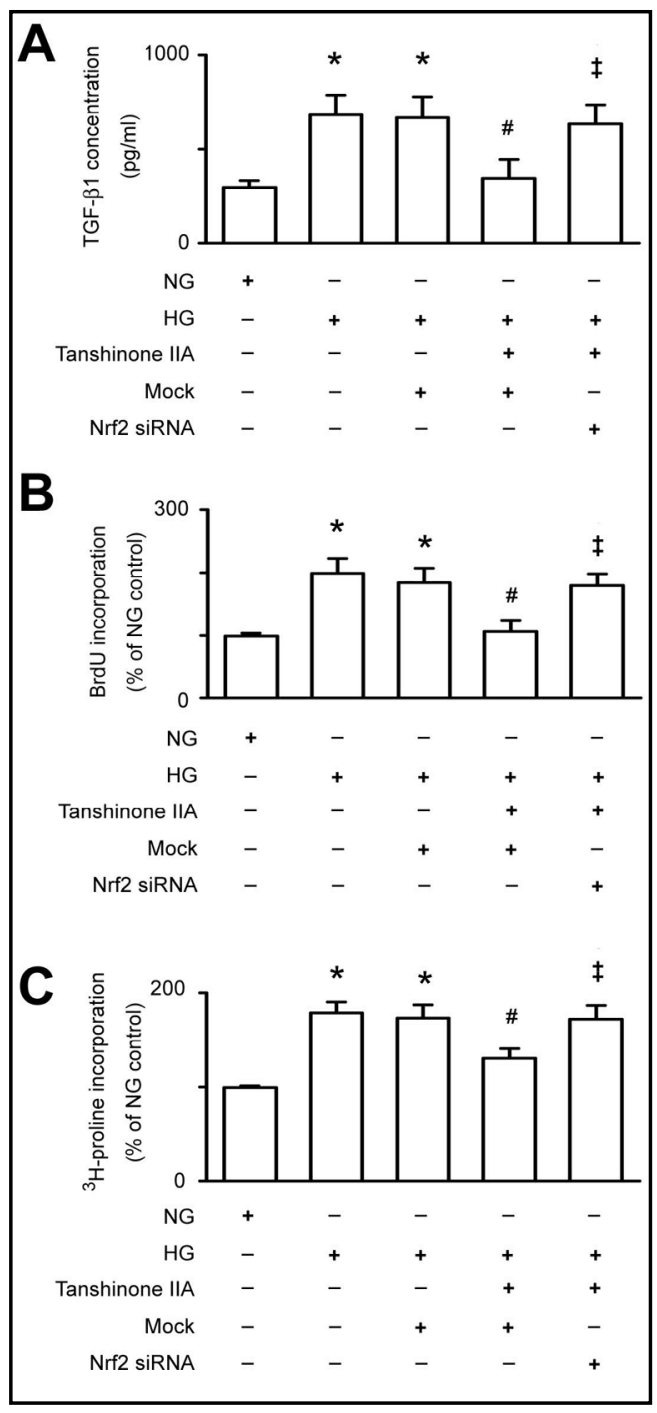

Fig. 5. Tanshinone IIA inhibits high glucose induced TGF- $\beta 1$ expression, cell proliferation and collagen synthesis in cardiac fibroblasts in Nrf2 dependent manner. Transfected cells were pretreated with $10 \mu \mathrm{M}$ tanshinone IIA for $12 \mathrm{~h}$ then cultured in high glucose (HG) medium for $24 \mathrm{~h}$. Results are expressed as means \pm S.E.M. $(\mathrm{n}=6) . *$ indicates a $\mathrm{P}$ value $<0.05$ compared with the normal glucose (NG) control group. \# indicates a $P$ value $<0.05$ compared with the HG group. $\neq$ indicates a $P$ value $<0.05$ compared with the tanshinone IIA treatment in control siRNAtransfected mock HG group. (A) Nrf2 siRNA prevents the inhibitory effect of tanshinone IIA on HG-induced TGF- $\beta 1$ expression. (B) Nrf2 siRNA prevents the inhibitory effect of tanshinone IIA on HG-induced cell proliferation. (C) Nrf2 siRNA prevents the inhibitory effect of tanshinone IIA on HG-induced collagen synthesis. 
this study further explored the possible mechanisms underlying tanshinone IIA inhibition of high glucose-induced fibroblast proliferation and collagen synthesis. In this study, we found high glucose led to increase cell proliferation, collagen synthesis, the expression of TGF- $\beta 1$ and Smad2/3 phosphorylation in cardiac fibroblasts, and this observation was consistent with the role of TGF- $\beta 1$ in promoting collagen synthesis, suggesting the presence of the TGF- $\beta 1 /$ Smad signaling pathway in cardiac fibroblasts. As a result, the cell proliferation and collagen synthesis in cardiac fibroblasts was attenuated. The results suggested that tanshinone IIA deactivated the high glucose-induced TGF- $\beta 1 /$ Smad signaling pathway by suppressing the expression of TGF- $\beta 1$ and phosphorylation of Smad2/3, hence, the synthesis of collagen in cardiac fibroblasts was correspondingly reduced. Therefore, the results of our present study implicates that tanshinone II is a potential target on new drug development against the illness of cardiac fibrosis in clinic. Low-dose tanshinone IIA pretreatment $(1 \mu \mathrm{M})$ had almost no impact on the high glucose induced expression of TGF- $\beta 1$ and Smad2/3 phosphorylation, but $10 \mu \mathrm{M}$ and $30 \mu \mathrm{M}$ tanshinone IIA pretreatment significantly reduced the high glucose induced expression of TGF- $\beta 1$ and Smad2/3 phosphorylation. These results suggest tanshinone IIA may prevent high glucose induced cell proliferation and collagen production by inhibiting TGF- $\beta 1-S m a d 2 / 3$ signaling. This mechanism is similar to that demonstrated in other previous studies, in which tanshinone IIA prevented myocardial fibrosis [23]. In addition, TGF- $\beta 1$ signal transduction is very complex [18]. TGF- $\beta 1$ is one of many proteins involved in modifying the extracellular matrix [18]. Indeed, the interplay between the extracellular matrix, cardiomyocytes, fibroblasts and the key signaling proteins involved remains an area of intense research [24]. The results of this study revealed tanshinone IIA can inhibit high glucose-induced TGF- $\beta 1$ expression and Smad2/3 phosphorylation, but the precise cellular target of tanshinone IIA remains unclear, and these mechanisms require further investigation.

Despite clearly acting as an inhibitor of the high glucose-induced fibroblast proliferation, collagen synthesis, and the expression of TGF- $\beta 1$, tanshinone IIA has been shown to demonstrate anti-oxidant [5] and anti-inflammatory effects [4, 25]. It has been suggested oxidative stress plays a critical role in inducing cardiac fibrosis in chronic diabetes [11]. ROS have been shown to promote the development of fibrosis through activating latent TGF- $\beta$ [11]. As a result, we cannot definitely exclude other potential mechanisms for the effects observed in vitro. Whilst we focused upon modification of ROS levels as a mediator of downstream high glucose-induced signaling, in this study, we found tanshinone IIA treatment alleviated the production of ROS in cultured cardiac fibroblasts. In addition, tanshinone IIA blocked high glucose promoted induced cardiac fibroblast proliferation and collagen synthesis via inhibition of ROS. To examine the role of oxidative stress in high glucose-induced cardiac fibroblast activation, we treated cardiac fibroblasts with the antioxidant NAC. NAC is a thiol-containing radical scavenger and glutathione precursor [26, 27]. Similarly, antioxidant treatment using NAC also attenuated high glucose-induced increases in ROS production, fibroblast proliferation and collagen synthesis in cultured cardiac fibroblasts. These results further strengthen the role of tanshinone IIA as an antioxidant by inhibiting oxidative stress. However, whether any antioxidant could have the beneficial effects of tanshinone IIA in decreasing high glucose-induced cardiac fibroblast activation remains to be examined in future study. On the other hand, Nrf2, a major regulator of the antioxidant response elements, has long been known for resisting oxygen-free radicals and reducing oxidative stress in alleviating of cardiac fibrosis [28]. Our study shows tanshinone IIA can not only ameliorate high glucose-induced oxidative stress, but also effectively promote Nrf2 translocation to the nucleus, which in turn up-regulated HO-1. Our previous study confirmed tanshinone IIA regulates Nrf2 activation and HO-1 expression in vascular endothelial cells [4]. The generation of HO-1 has been widely recognized as an effective cellular strategy to respond a variety of cellular impacts, such as inflammation and oxidative challenges. The HO-1 induced effects may be mediated by multiple functions of HO-1. For example, the antifibrotic effects of HO-1 was reported caused by its degradation of heme, resulting in the liberation of equimolar amounts of iron, carbon monoxide (CO) and biliverdin [29]. Although 


\section{Cellular Physiology Cell Physiol Biochem 2018;51:2250-2261

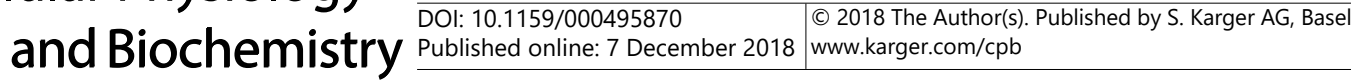 \\ Tsai et al.: Tanshinone IIA Inhibits Collagen Synthesis}

the exact mechanisms involved in anti-fibrotic effects of HO-1 have not been fully elucidated, Tanshinone IIA, as confirmed in this study, can induce the expression of HO-1 in cardiac fibroblasts, the observed anti-fibrotic effects of tanshinone IIA might be mediated, at least in part, by one or more of HO-1 by-products. However, whether inhibition of HO-1 activity could block tanshinone IIA-induced anti-fibrotic action to suggest the role of HO-1 on the anti-fibrotic effect of tanshinone IIA in cardiac fibroblasts remains to be elucidated.

Moreover, the impacts of tanshinone IIA on Nrf2 signaling in cardiac fibroblasts under high glucose conditions were unclear. In this study, by using an Nrf2 siRNA, we confirmed the anti-fibrotic effects of tanshinone IIA on cardiac fibroblasts under high glucose conditions were Nrf2 dependent. Nrf2 was dissociated with Keapl following ROS generation and translocated into the nucleus to bind the antioxidant response elements, then, initiated the transcription of antioxidant genes such as HO-1, superoxide dismutase, and glutathione to defend the ROS productions [28]. Interestingly, Nrf2 has been reported to attenuate dystrophic muscle fibrosis through inhibiting the TGF- $\beta 1 /$ Smad Pathway [30]. The present study used siRNA to inhibit Nrf2 expression, and demonstrated Nrf2 silencing significantly increased the expression of TGF- $\beta 1$. Thus, tanshinone IIA activated Nrf2 signaling might reduce high glucose-induced cell proliferation and collagen synthesis through direct anti-fibrotic effects of Nrf2 and indirect down-regulation of ROS levels. However, the exact molecular mechanism of Nrf2 regulation by tanshinone IIA remains unknown and warrants further investigation. Diabetes-associated cardiac fibrosis is a severe cardiovascular pathological change. This study demonstrated Tanshinone IIA could inhibit fibroblast activation in cell study. While these results are promising, a lot more work needs to be done in elucidating the signal pathways involved in the action of tanshinone IIA on these cells. Moreover, in vivo experiments in animal models are essential to establish the validity of these in vitro results in the future.

\section{Conclusion}

Based on this study and studies published by other groups, we propose tanshinone IIA via Nrf2 activation can partially block intracellular TGF- $\beta 1$ signal transduction and inhibit the proliferation and collagen synthesis in cardiac fibroblasts. In this way, tanshinone IIA reduces collagen synthesis and TGF- $\beta 1$ expression induced by high glucose, suggesting it may have some clinical application in preventing and treating diabetic myocardial fibrosis. In conclusion, our study demonstrated tanshinone IIA attenuates high glucose induced collagen synthesis, and it seems the anti-fibrotic effects of the tanshinone IIA, at least in part, occur by reducing the expression of the profibrogenic cytokine TGF- $\beta 1$ and inhibiting TGF- $\beta 1-S m a d 2 / 3$ signaling. These findings suggested tanshinone IIA might have therapeutic potential in treating diabetes-induced cardiac fibrosis.

\section{Acknowledgements}

This work was supported in part by Ministry of Science and Technology Grants (Grant No.: MOST 106-2314-B-016 -037 -MY3, MOST 106-2320-B-016-003-MY2), National Defense Medical Center (Grant No: MAB-106-033), Teh-Tzer Study Group for Human Medical Research Foundation (Grant No: A1061037; A1061054), and Tri-Service General Hospital Grants (Grant No: TSGH-C105-028; C106-021; C108-008-S04), Taipei, Taiwan, R.O.C.

Conception and design: YTT, SHL, and CST; executing experiment: YTT, and THC; analysis and interpretation: YTT, SHL, SPL, YLC, CYL, and THC; drafting the manuscript for important intellectual content: SPL, YTT and SHL; final approval of the manuscript: YTT, SHL, THC, SPL, YLC and CST. All authors read and approved the final manuscript. 


\section{Cellular Physiology Cell Physiol Biochem 2018;51:2250-2261

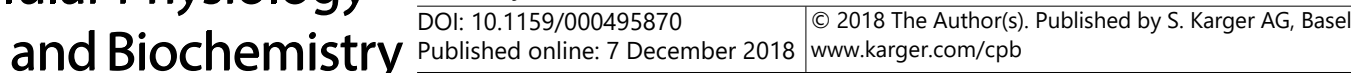 \\ Tsai et al.: Tanshinone IIA Inhibits Collagen Synthesis}

The in vivo experiment conformed to the Guide for the Care and Use of Laboratory Animals published by the US National Institutes of Health (NIH Publication No. 85-23, revised 1996) and was also approved by the Institutional Animal Care and Use Committee of China Medical University (IACUC 2016-032).

\section{Disclosure Statement}

The authors declare that there is no conflict of interests regarding the publication of this paper.

\section{References}

1 Akaberi M, Iranshahi M, Mehri S: Molecular Signaling Pathways Behind the Biological Effects of Salvia Species Diterpenes in Neuropharmacology and Cardiology. Phytother Res 2016;30:878-893.

$>2$ Hong HJ, Liu JC, Chen PY, Chen JJ, Chan P, Cheng TH: Tanshinone IIA prevents doxorubicin-induced cardiomyocyte apoptosis through Akt-dependent pathway. Int J Cardiol 2012;157:174-179.

3 Chan P, Chen YC, Lin LJ, Cheng TH, Anzai K, Chen YH, Liu ZM, Lin JG, Hong HJ: Tanshinone IIA Attenuates $\mathrm{H}(2) \mathrm{O}(2)$-induced injury in human umbilical vein endothelial cells. Am J Chin Med 2012;40:1307-1319.

4 Zhuang S, Cheng TH, Shih NL, Liu JC, Chen JJ, Hong HJ, Chan P: Tanshinone IIA Induces Heme Oxygenase 1 Expression and Inhibits Cyclic Strain-Induced Interleukin 8 Expression in Vascular Endothelial Cells. Am J Chin Med 2016;44:377-388.

5 Chan P, Liu JC, Lin LJ, Chen PY, Cheng TH, Lin JG, Hong HJ: Tanshinone IIA inhibits angiotensin II-induced cell proliferation in rat cardiac fibroblasts. Am J Chin Med 2011;39:381-394.

6 Mizamtsidi M, Paschou SA, Grapsa J, Vryonidou A: Diabetic cardiomyopathy: a clinical entity or a cluster of molecular heart changes? Eur J Clin Invest 2016;46:947-953.

7 Russo I, Frangogiannis NG: Diabetes-associated cardiac fibrosis: Cellular effectors, molecular mechanisms and therapeutic opportunities. J Mol Cell Cardiol 2016;90:84-93.

>8 Shen N, Li X, Zhou T, Bilal MU, Du N, Hu Y, Qin W, Xie Y, Wang H, Wu J, Ju J, Fang Z, Wang L, Zhang Y: Shensong Yangxin Capsule prevents diabetic myocardial fibrosis by inhibiting TGF-beta1/Smad signaling. J Ethnopharmacol 2014;157:161-170.

-9 Cheng TH, Cheng PY, Shih NL, Chen IB, Wang DL, Chen JJ: Involvement of reactive oxygen species in angiotensin II-induced endothelin-1 gene expression in rat cardiac fibroblasts. J Am Coll Cardiol 2003;42:1845-1854.

10 Euler G: Good and bad sides of TGFbeta-signaling in myocardial infarction. Front Physiol 2015;6:66.

11 Liu C, Lu XZ, Shen MZ, Xing CY, Ma J, Duan YY, Yuan LJ: N-Acetyl Cysteine improves the diabetic cardiac function: possible role of fibrosis inhibition. BMC Cardiovasc Disord 2015;15:84.

$>12$ Li J, Ichikawa T, Villacorta L, Janicki JS, Brower GL, Yamamoto M, Cui T: Nrf2 protects against maladaptive cardiac responses to hemodynamic stress. Arterioscler Thromb Vasc Biol 2009;29:1843-1850.

13 Wu J, Xia S, Kalionis B, Wan W, Sun T: The role of oxidative stress and inflammation in cardiovascular aging. Biomed Res Int 2014;2014:615312.

-14 Shamhart PE, Luther DJ, Adapala RK, Bryant JE, Petersen KA, Meszaros JG, Thodeti CK: Hyperglycemia enhances function and differentiation of adult rat cardiac fibroblasts. Can J Physiol Pharmacol 2014;92:598-604.

15 Aguilar H, Fricovsky E, Ihm S, Schimke M, Maya-Ramos L, Aroonsakool N, Ceballos G, Dillmann W, Villarreal F, Ramirez-Sanchez I: Role for high-glucose-induced protein 0-GlcNAcylation in stimulating cardiac fibroblast collagen synthesis. Am J Physiol Cell Physiol 2014;306:C794-804.

-16 Fiaschi T, Magherini F, Gamberi T, Lucchese G, Faggian G, Modesti A, Modesti PA: Hyperglycemia and angiotensin II cooperate to enhance collagen I deposition by cardiac fibroblasts through a ROS-STAT3dependent mechanism. Biochim Biophys Acta 2014;1843:2603-2610.

17 Ziyadeh FN, Sharma K, Ericksen M, Wolf G: Stimulation of collagen gene expression and protein synthesis in murine mesangial cells by high glucose is mediated by autocrine activation of transforming growth factorbeta. J Clin Invest 1994;93:536-542. 


\section{Cellular Physiology Cell Physiol Biochem 2018;51:2250-2261

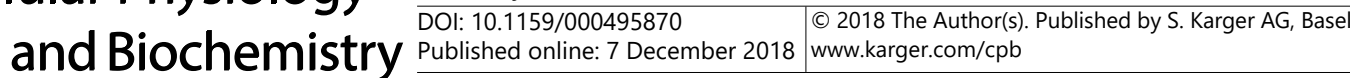 \\ Tsai et al.: Tanshinone IIA Inhibits Collagen Synthesis}

18 Meng XM, Nikolic-Paterson DJ, Lan HY: TGF-beta: the master regulator of fibrosis. Nat Rev Nephrol 2016;12:325-338.

19 Cao L, Huang B, Fu X, Yang J, Lin Y, Lin F: Effects of tanshinone IIA on the regulation of renal proximal tubular fibrosis. Mol Med Rep 2017;15:4247-4252.

20 Li LC, Kan LD: Traditional Chinese medicine for pulmonary fibrosis therapy: Progress and future prospects. J Ethnopharmacol 2017;198:45-63.

-21 Yang F, Li P, Li H, Shi Q Li S, Zhao L: microRNA-29b Mediates the Antifibrotic Effect of Tanshinone IIA in Postinfarct Cardiac Remodeling. J Cardiovasc Pharmacol 2015;65:456-464.

-22 Shu M, Hu XR, Hung ZA, Huang DD, Zhang S: Effects of tanshinone IIA on fibrosis in a rat model of cirrhosis through heme oxygenase-1, inflammation, oxidative stress and apoptosis. Mol Med Rep 2016;13:30363042.

23 Zhan CY, Tang JH, Zhou DX, Li ZH: Effects of tanshinone IIA on the transforming growth factor beta1/Smad signaling pathway in rat cardiac fibroblasts. Indian J Pharmacol 2014;46:633-638.

24 Khan R, Sheppard R: Fibrosis in heart disease: understanding the role of transforming growth factor-beta in cardiomyopathy, valvular disease and arrhythmia. Immunology 2006;118:10-24.

25 Gao S, Liu Z, Li H, Little PJ, Liu P, Xu S: Cardiovascular actions and therapeutic potential of tanshinone IIA. Atherosclerosis 2012;220:3-10.

26 Ezerina D, Takano Y, Hanaoka K, Urano Y, Dick TP: N-Acetyl Cysteine Functions as a Fast-Acting Antioxidant by Triggering Intracellular H2S and Sulfane Sulfur Production. Cell Chem Biol 2018;25:447-459.e444.

27 Samuni Y, Goldstein S, Dean OM, Berk M: The chemistry and biological activities of N-acetylcysteine. Biochim Biophys Acta 2013;1830:4117-4129.

28 Barancik M, Gresova L, Bartekova M, Dovinova I: Nrf2 as a key player of redox regulation in cardiovascular diseases. Physiol Res 2016;65 Suppl 1:S1-s10.

-29 Loboda A, Jozkowicz A, Dulak J: HO-1/CO system in tumor growth, angiogenesis and metabolism Targeting HO-1 as an anti-tumor therapy. Vascul Pharmacol 2015;74:11-22.

30 Sun C, Li S, Li D: Sulforaphane mitigates muscle fibrosis in mdx mice via Nrf2-mediated inhibition of TGFbeta/Smad signaling. J Appl Physiol (1985) 2016;120:377-390. 